\title{
PERSPECTIVES
}

OPINION

\section{Antibodies and tuberculosis: finally coming of age?}

\section{Hao Li and Babak Javid (10}

Abstract | Are antibodies important for protection against tuberculosis? The jury has been out for more than 100 years. B cell depletion in experimental Mycobacterium tuberculosis infection failed to identify a major role for these cells in immunity to tuberculosis. However, recent identification of naturally occurring antibodies in humans that are protective during $M$. tuberculosis infection has reignited the debate. Here, we discuss the evidence for a protective role for antibodies in tuberculosis and consider the feasibility of designing novel tuberculosis vaccines targeting humoral immunity.

The role of humoral immunity in tuberculosis (TB) is controversial. Almost all clinical and preclinical vaccine candidates for TB that are in development target cell-mediated immunity ${ }^{1}$. As an intracellular pathogen, it has been assumed that Mycobacterium tuberculosis, which is the causative agent of TB, would be inaccessible to circulating antibodies; however, cells infected with $M$. tuberculosis would be vulnerable to killing by the effector arm of the cell-mediated immune response. Several publications have stimulated a re-evaluation of the contribution of antibody-mediated immunity to protection against $M$. tuberculosis ${ }^{2-5}$, but a number of important questions remain. Why has antibody-mediated immunity been largely overlooked for vaccine development? Which approaches led to the recent series of high-profile papers re-examining the role of antibodies in human immunity to TB? How do antibodies offer protection against $M$. tuberculosis? As the only licensed vaccine for TB, bacillus Calmette-Guerin (Mycobacterium bovis-BCG or BCG) offers highly variable protection against $\mathrm{TB}^{6}$ and almost no protection against the transmissible form of the disease, there is an urgent need for a new TB vaccine if we are to interrupt the current TB emergency; in 2016 M. tuberculosis claimed more lives than any other single pathogen ${ }^{7}$. In this Opinion piece, we consider whether antibody-based vaccines could show promise as a new vaccine strategy in $\mathrm{TB}$ and discuss the key questions that still need to be addressed in this field.

\section{Antibodies in tuberculosis} Why is antibody-mediated immunity controversial? Koch's 1882 discovery of M. tuberculosis as the causative agent of TB stimulated an interest in specific therapies, including serum therapy, which had shown considerable success in the treatment of other infectious diseases (reviewed in REF. ${ }^{8}$ ). However, dozens of studies using serum therapy in TB showed highly variable results, partly owing to differences in antigens, antibody titres in serum and host animals (reviewed in REF. ${ }^{9}$ ). As such, interest in serum therapy waned rapidly and came to a halt following the more promising discoveries of the BCG vaccine strain and streptomycin, the first anti-tubercular antibiotic.

There was also increasing evidence for the importance of cell-mediated responses in protection against TB. Mice lacking key elements of cell-mediated immunity, such as IFN $\gamma$, tumour necrosis factor (TNF) or T cells, and patients with HIV are highly susceptible to $\mathrm{TB}^{10,11}$. This contrasted with the relatively modest disease phenotypes seen when mice lacking B cells were infected with $M$. tuberculosis ${ }^{12,13}$. It was shown in experimentally infected cynomolgus macaques that $M$. tuberculosis-containing granulomata are surrounded by B cell clusters that express proliferation-associated markers and secrete $M$. tuberculosis-specific antibodies ${ }^{14}$. However, depletion of B cells using the therapeutic monoclonal antibody rituximab resulted in highly heterogeneous effects in local granuloma immune modulation, perhaps owing to the variety of antibody and non-antibody functions of B cells, but had no effect on disease outcome ${ }^{15}$.

While these studies did not by themselves prove a lack of B cell or antibody involvement in immunity to M. tuberculosis, they did cast doubt on the potential importance of B cells and antibodies in animal models of TB infection. In parallel, however, studies using antibodies in passive immunization suggested that certain antibodies are protective against TB. Administration of monoclonal antibodies raised against $M$. tuberculosis antigens or pooled mouse or human immunoglobulins could ameliorate disease in animal models in most ${ }^{16-23}$, although not all ${ }^{24}$, cases. Perhaps owing to caution in interpretation of some of the data - antibodies mixed with $M$. tuberculosis in vitro before infection, as in some of the experiments, might have induced clumping and exaggerated the degree of offered protection - there was greater enthusiasm for the development of recombinant vaccines that elicited potent cell-mediated immune responses in animal models.

Antibodies back in vogue. In 2013, the first late-stage clinical trial in the modern era for a new vaccine against TB was reported; disappointingly, it showed no efficacy over and above routine BCG immunization ${ }^{25}$. The vaccine, known as MVA85A, delivered a recombinant immunodominant epitope, antigen $85 \mathrm{~A}$, via modified vaccinia Ankara virus. MVA85A performed as engineered: it elicited a potent response of $\mathrm{CD} 4^{+} \mathrm{T}$ cells secreting IFN $\gamma^{25,26}$. Therefore, its failure (though unsurprising ${ }^{27}$ to some) led to a re-evaluation of what may constitute a protective immune response against M. tuberculosis. Some questioned whether IFN $\gamma$-secreting $\mathrm{CD} 4^{+} \mathrm{T}$ cells were the key to cell-mediated immunity ${ }^{28}$, and there was also renewed interest in the potential role, if any, of antibody-mediated immunity in M. tuberculosis infection. 
Important lessons had been learnt in the interim. The focus shifted to studying naturally elicited human antibody responses. It was also clear from the renaissance in broadly neutralizing antibodies in the HIV field that not all HIV-infected individuals made these protective antibody responses ${ }^{29}$. As such, pooling antibodies from multiple donors might drown out signals of protection within the noise of the majority of donors that did not make protective responses. Below, we focus on four key papers published since 2016 that applied these principles to advance the field ${ }^{2-5}$.

A low titre of antibodies against lipoarabinomannan (LAM), which is a key glycolipid found on the surface of Mycobacterium spp., had been associated with disseminated TB in children ${ }^{30}$, and passive immunization of mice with monoclonal antibodies against the sugar component of LAM, arabinomannan, had been demonstrated to be protective in mouse infection models ${ }^{19,21}$. Achkar and colleagues obtained serum from 30 healthy volunteers immunized with BCG, which stimulates both cell-mediated and humoral immunity ${ }^{2}$. They demonstrated that BCG elicited antibody responses against both arabinomannan and LAM and that post-vaccination serum was able to opsonize M. tuberculosis for phagocytosis by macrophages, enhance phagolysosome fusion and inhibit intracellular growth of M. tuberculosis. Importantly, the titre of arabinomannan-specific responses in individuals correlated with the magnitude of these protective responses ${ }^{2}$.

Alter and Fortune examined polyclonal serum from 20 patients with active TB and 22 individuals with latent TB infection (LTBI) - these are individuals with immunological evidence of exposure to M. tuberculosis but with no symptoms or signs of active infection ${ }^{4}$. They used a 'systems serology' approach to interrogate approximately 70 features of antibody function that are not associated with the variable domain of the antibody molecule. Of these features, 19 differed significantly between antibodies derived from South African patients with LTBI and those derived from South African patients with active $\mathrm{TB}$, and 9 of these features were sufficient to distinguish between the two groups. Some of the features, in particular, glycosylation status of the crystallizable fragment $(\mathrm{Fc})$ portion of the antibody molecule, were also validated in samples from Texas, USA, and Mexico. In experiments where human macrophages were infected with M. tuberculosis, antibodies from the individuals with LTBI

were better at restricting intracellular growth of the pathogen than antibodies from patients with active $\mathrm{TB}^{4}$.

Our own group and the groups of Kaufmann and Wardemann chose to compare antibody responses between patients with active TB and health-care workers (HCWs) who had potential occupational exposure to M. tuberculosis ${ }^{3,5}$. Zimmermann et al. identified M. tuberculosisspecific antibody responses in both patients with active TB and HCWs. Approximately $40 \%$ of monoclonal antibodies isolated from the plasmablasts of these individuals recognized mycobacterial antigens, with the majority of $M$. tuberculosis-specific antibodies targeting surface-exposed antigens: either binding intact bacteria or surface antigens ${ }^{5}$. It was found that 7 of 12 isolated immunoglobulin A ( $\operatorname{IgA})$ monoclonal antibodies but 0 of 16 isolated IgG monoclonal antibodies were able to restrict $M$. tuberculosis growth within the lung epithelial cell line A549. However, although HCWs had slightly higher titres of M. tuberculosis-specific IgA, both restrictive and ineffective $M$. tuberculosis-specific monoclonal antibodies were isolated from patients with active TB and the healthy HCWs. Therefore, it could not be inferred from these studies how important antibodies were for protection from active TB.

It is difficult to control for relative exposure to M. tuberculosis when studying protective immunity, which can confound true immunity from lack of exposure. Our subjects were from the Beijing TB hospital, which treats thousands of patients with TB annually. Unlike in more developed economies, HCWs in China rarely use adequate personal protective equipment when encountering patients with TB. Therefore, HCWs have a substantial M. tuberculosis exposure burden, and many show evidence of LTBI or develop active $\mathrm{TB}^{31,32}$, but many others remain healthy, with no evidence of LTBI. We isolated polyclonal immunoglobulin from patients with TB and HCWs - some of whom had LTBI but also some who were highly exposed but uninfected (HEBUI). We tested antibodies from single donors in two models: one examined relative protection from M. tuberculosis in a mouse model of aerosol infection, and one examined protection in an in vitro human whole blood assay. Antibodies from a subset of both donors with LTBI and HCWs who were HEBUI (7 of 48) restricted $M$. tuberculosis growth in both assays. Those donors (who were
HEBUI) with no evidence of prior infection by $M$. tuberculosis may represent human 'restrictors' that can control M. tuberculosis infection - although an alternative explanation might be that current tests for LTBI merely fail to identify the mediators of immune exposure in these individuals ${ }^{33}$. By contrast, no patients with active TB made protective antibody responses ${ }^{3}$. Intriguingly, $M$. tuberculosis growth restriction owing to antibodies from protective donors was completely abrogated both in vivo and in vitro in the absence of $\mathrm{CD} 4^{+} \mathrm{T}$ cells and required formation of immune complexes for full efficacy, suggesting that protection was not due simply to neutralization of M. tuberculosis by antibodies ${ }^{3}$.

Together, these studies revived interest in humoral immunity to TB and suggested that subjects with high exposure to M. tuberculosis but without active TB represent the ideal population in which to study and isolate protective antibodies.

One further study deserves highlighting. Unlike the preceding four studies, it focused on BCG-elicited immunity in murine TB and did not involve human antibodies. Casadevall and colleagues showed that when mice were immunized with BCG grown with an intact capsule, they were better able to resist subsequent infection with pathogenic M. tuberculosis ${ }^{34}$. Importantly, encapsulated BCG was not only better at eliciting higher titres of class-switched antibody responses, it was also more effective at generating IFN $\gamma$ and poly-functional $\mathrm{T}$ cell responses, which together led to an order of magnitude lower bacterial burden ${ }^{34}$.

\section{Antibody-mediated protection}

How do antibodies protect against TB? As already mentioned above, what is clear is that protection appears to be more complex than simple neutralization of bacteria and requires cell-mediated immune functions. For example, macrophages, immune complex formation (FIG. 1) and T cell responses have all been proposed to be involved (FIG. 2) - although evidence for many of these mechanisms is still highly preliminary.

\section{What are the protective antigens?}

The $M$. tuberculosis genome encodes approximately 4,000 genes ${ }^{35}$, and the function and subcellular localization of the products of many of these genes remain unknown. Furthermore, M. tuberculosis has a complex glycolipid and glycoprotein capsule ${ }^{36}$, which would represent the surface of the pathogen in natural infection. To complicate matters, for pragmatic purposes, $M$. tuberculosis is 


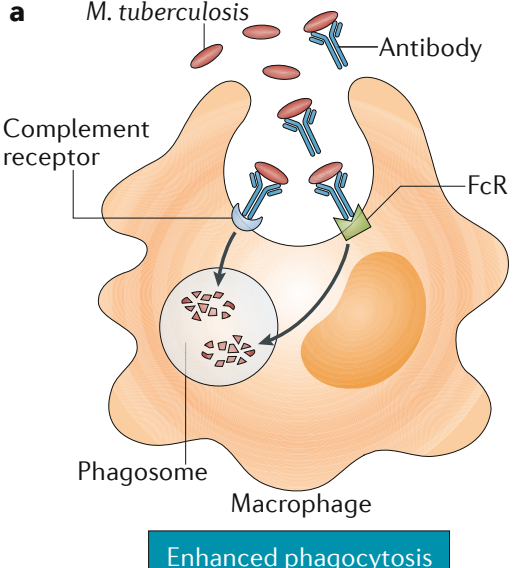

C

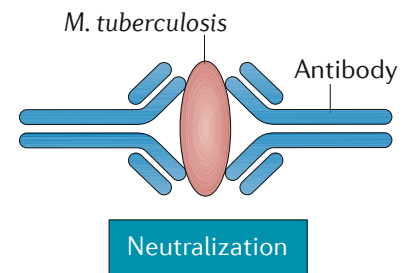

b

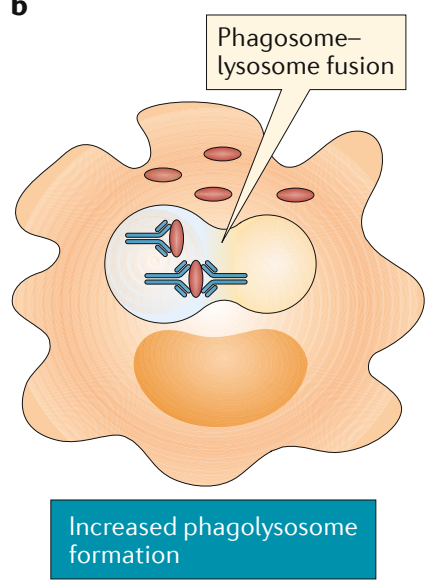

d

e

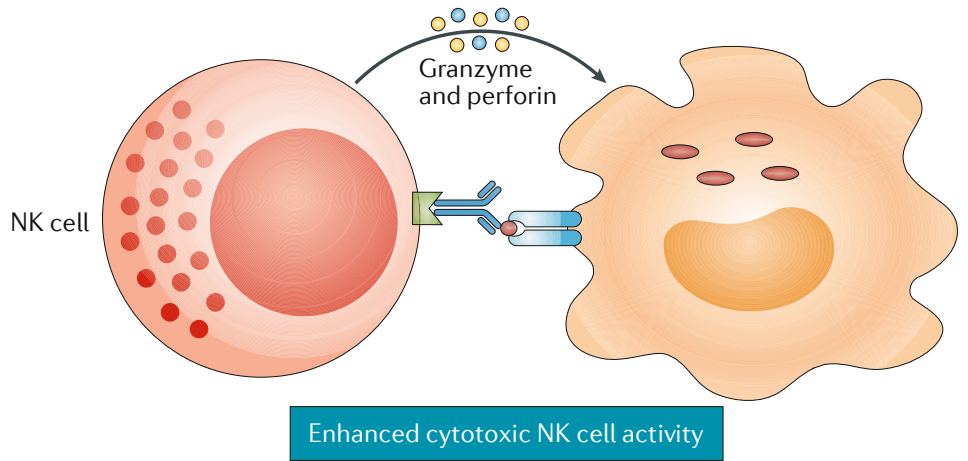

Fig. 1 | Potential mechanisms of antibody-mediated protection against Mycobacterium tuberculosis. The figure indicates the different ways in which antibodies may enhance immunity to Mycobacterium tuberculosis. a|Complexes of $M$. tuberculosis bound to antibody can be more readily phagocytosed by macrophages via crystallizable fragment $\left(\mathrm{F}_{\mathrm{c}}\right)$ receptors $(\mathrm{FcRs})$ and complement receptors. $\mathbf{b}$ | Antibodies specific for M. tuberculosis can increase phagosome-lysosome fusion - which M. tuberculosis usually interferes with - and thereby restrict the growth of $M$. tuberculosis. $\mathbf{c} \mid$ Antibodies against $M$. tuberculosis may have direct microbicidal or neutralizing activity, or they may prevent the uptake of the bacteria by cells not expressing the appropriate FcRs to promote M. tuberculosis killing. d) Antibodies against $M$. tuberculosis have been shown to promote inflammasome activation in macrophages, which is associated with ASC speck formation and IL-1 $\beta$ secretion. e $\mid$ Antibodies specific for M. tuberculosis may stimulate killing of M. tuberculosis-infected cells via natural killer (NK) cell-mediated antibody-dependent cell cytotoxicity. It should be noted that there is only limited evidence for some of these mechanisms.

routinely grown in the laboratory in media containing mild detergent, which strips off much of the capsule ${ }^{36}$.

As already mentioned, immunization of mice with BCG grown with an intact capsule offered more robust protection against M. tuberculosis ${ }^{34}$. Protective antibody responses from HCWs were significantly enhanced when assessed against capsuleintact M. tuberculosis. Furthermore, depletion of antibodies targeting soluble antigens from $M$. tuberculosis failed to remove the protective fraction, whereas the depletion of antibodies targeting intact $M$. tuberculosis abrogated protection. Together, these data strongly suggested that protective antibodies were directed against surface-displayed capsule antigens ${ }^{3}$. However, what was the nature of these antigens? Panning an M. tuberculosis proteome array ${ }^{37}$ failed to identify potential protective antigens ${ }^{3}$. Possibly, the majority of protective responses were directed against non-protein antigens, and indeed carbohydrate-protein conjugate vaccines against arabinomannan ${ }^{38}$ and a peptide mimotope vaccine targeting $\mathrm{LAM}^{39}$ have shown efficacy in mouse models. But while there is a clear correlation between arabinomannan antibody titres and protection ${ }^{2,30}$, only one study to date has isolated naturally occurring human monoclonal antibodies against M. tuberculosis ${ }^{5}$. The antigenic targets of most of the human antibodies that recognize M. tuberculosis have not been identified, and although some antibodies were shown to bind the cell surface epitopes LAM and heparin-binding haemagluttinin ${ }^{20}$, antibodymediated protection in this study appeared to be independent of these antigens ${ }^{5}$.

\section{Roles of invariant antibody functions.}

Although antibody-mediated immunity to viruses involves simple neutralization, broadly neutralizing antibodies to HIV also require antibody Fc-mediated functions for in vivo efficacy ${ }^{40}$. Systems serology identified a number of Fc-mediated antibody functions: principally, glycosylation of the Fc molecule, which had previously been demonstrated to be important for protection of passive antibody transfer in mice ${ }^{41}$, but immune complex and inflammasome activation functions were also identified as being strongly associated with protective responses derived from LTBI donors ${ }^{4}$.

$M$. tuberculosis has adapted to an intracellular lifestyle - therefore, the first step of productive infection is phagocytosis by a host cell. The potential protective role for opsonophagocytosis by antibody is therefore less straightforward ${ }^{42}$ than that for extracellular pathogens such as pneumococcal species, where it plays a clearly protective role ${ }^{43}$. The intriguing finding that the $\operatorname{IgA} 1$ isoform of a particular monoclonal antibody was protective, whereas the IgG1 isoform of the same monoclonal antibody was permissive for growth, in lung epithelial cells suggested that opsonizing antibodies in TB act as 'trojan horses' (REF.5). In support of this idea, 
a

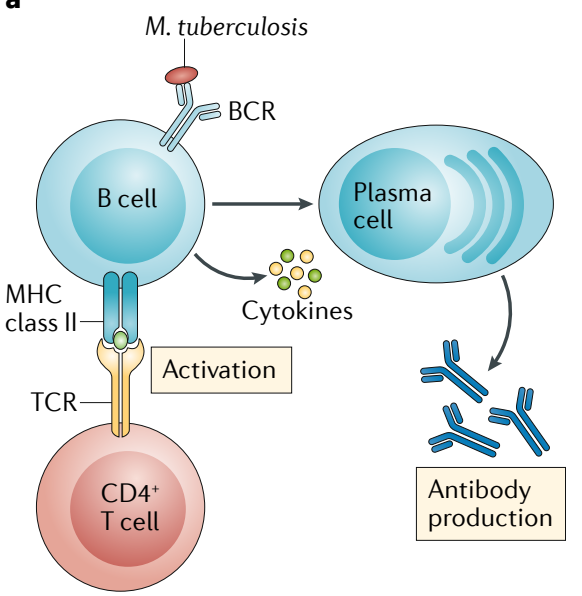

b

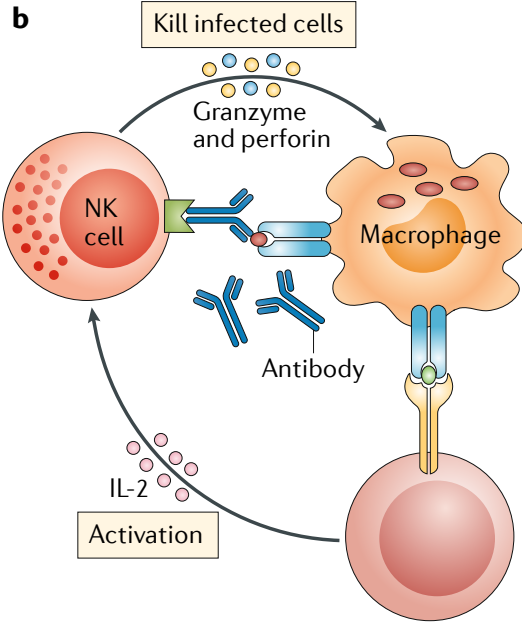

C

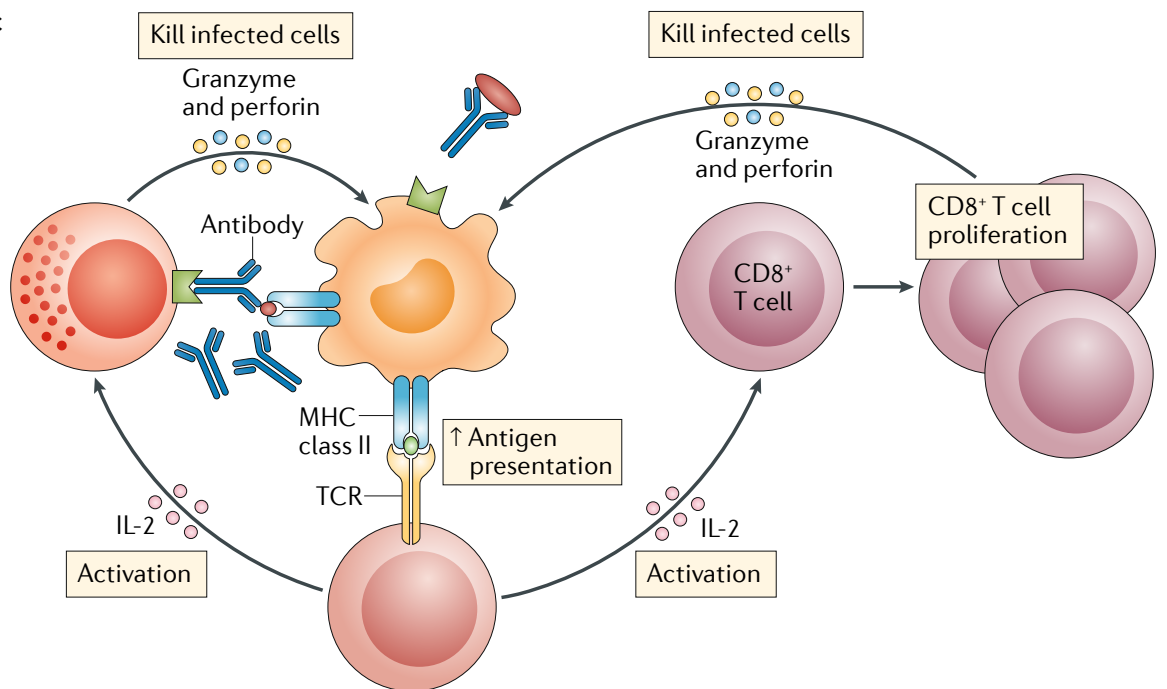

Fig. 2 | Potential CD4 ${ }^{+}$T cell-dependent mechanisms of antibody-mediated protection. There are several ways in which CD4 ${ }^{+} \mathrm{T}$ cells and antibodies may cooperate to promote immunity to Mycobacterium tuberculosis. a|CD4 ${ }^{+} \mathrm{T}$ cells can provide help to $\mathrm{B}$ cells to stimulate the maturation of antibody responses. $\mathbf{b} \mid \mathrm{CD} 4^{+} \mathrm{T}$ cells may stimulate antibody-mediated killing of $M$. tuberculosisinfected cells indirectly by producing cytokines that can activate natural killer (NK) cells and enhance antibody-dependent cell cytotoxicity responses. $\mathbf{c}$ |Complexes of $M$. tuberculosis and antibody may result in increased processing and presentation of $M$. tuberculosis antigens to $C D 4^{+} T$ cells by professional phagocytes (for example, macrophages or dendritic cells). This can result in increased activation of helper $\mathrm{CD} 4^{+} \mathrm{T}$ cells that in turn promote cytotoxic $\mathrm{CD} 8^{+} \mathrm{T}$ cell and $\mathrm{NK}$ cell responses to enhance M. tuberculosis killing. BCR, B cell receptor; TCR, T cell receptor.

the relevant lung epithelial cells expressed neonatal Fc receptors, which bound only IgG and not IgA. Therefore, the protective role of IgA may have been due to binding and inhibition of M. tuberculosis phagocytosis by epithelial cells ${ }^{5}$. D'Arcy Hart's classic observation that opsonizing antibodies may also promote phagolysosomal fusion and killing by reactive oxygen species in macrophages ${ }^{44}$ may explain why some studies showed that opsonization was protective ${ }^{2,45}$ but others did not ${ }^{3,4}$.

A whole blood assay allowed us to interrogate mediators beyond single cells that were required for protection. Importantly, we identified a critical role for both immune complex function and $\mathrm{CD} 4^{+}$ $\mathrm{T}$ cells in antibody-mediated protection ${ }^{3}$. Antibodies play an important role for protection against another intracellular pathogen, Salmonella enterica subsp. enterica serovar Typhimurium ${ }^{16,46}$, for which a vaccine with $50-80 \%$ efficacy is commercially available. Although cellmediated immunity was sufficient for clearance of attenuated $S$. Typhimurium in a murine infection model, antibody was required for clearance of virulent
S. Typhimurium - specifically after vaccination $^{47}$. While we do not know precisely how $\mathrm{CD}^{+} \mathrm{T}$ cells and antibodies cooperate to protect against $M$. tuberculosis infection, there are several possibilities, including enhanced antigen presentation to $\mathrm{T}$ cells following phagocytosis of M. tuberculosis-antibody immune complexes or $\mathrm{CD} 4^{+} \mathrm{T}$ cell help for natural killer cell-mediated antibody-dependent cell cytotoxicity (FIG. 2).

\section{Antibody-based vaccines?}

It is now clear that naturally occurring human antibodies that are likely to be protective against $M$. tuberculosis infection exist in at least a subset of individuals. But can this observation lead to the development of a preventive vaccine? We first need to prioritize the characterization of the antigens that elicit protection, followed by the characterization of the molecular and cellular mechanisms mediating protection. This will be impossible without further studies of isolated protective monoclonal antibodies. However, even then, there are at least three further hurdles that need to be addressed. First, what are the in vitro correlates of in vivo protection? Second, are protective antibody responses of sufficient magnitude and efficacy to prevent disease? Third, can immunization with either recombinant antigens or a killed or attenuated mycobacterial strain elicit sufficiently protective antibodies in susceptible individuals?

In vitro correlates of protection. For many viral and bacterial infections, simple in vitro tests can predict in vivo protection by antibodies $^{48,49}$. However, for $M$. tuberculosis, a major bottleneck in vaccine development has been the total absence of any correlates for M. tuberculosis protection. Therefore, efficacy of vaccine candidates, even in non-human primates, cannot reliably predict protection in humans. As such, only late-stage clinical trials can properly test protection. This extremely costly option limits the potential number of candidates that can be tested ${ }^{1,50}$, and late-stage failures can influence funding of other candidates.

As already discussed, there are many potential mechanisms by which antibodies might mediate protection against M. tuberculosis - both antigen-dependent and antigen-independent (FICS. 1,2). But which should be used to test for protective antibody production? It is far from clear which of these mechanisms might be physiologically relevant, as in the example of 
opsonophagocytosis. Most groups have used M. tuberculosis cell-infection models to test antibody-mediated protection in vitro ${ }^{2,4,5}$, but this approach may miss more complex interactions that involve other components of the immune system ${ }^{3}$. Relatively unbiased approaches such as systems serology may be able to identify which antibody functions are most critical for protection - but lack of a preclinical gold standard will require validation in multiple models before the field can rely on in vitro correlates.

\section{Are antibodies sufficient for protection?}

The relative magnitude of $M$. tuberculosis growth restriction of naturally occurring human protective antibodies both in vivo and in vitro ${ }^{2-5}$ was less than one order of magnitude, raising questions about the physiological importance of antibodymediated protection. Furthermore, while one study used a clinical isolate of M. tuberculosis ${ }^{3,51}$, most studies have employed the laboratory-adapted strain H37Rv, and no studies have tested multiple clinical isolates. It is thought that natural transmission of $M$. tuberculosis is usually in microdroplets containing one bacillus ${ }^{52,53}$, while the typical murine challenge model infects mice with 100-200 bacilli per animal. In the HIV field, shifting to more physiological 'low-dose' challenge models resulted in more reliable assessment of vaccine candidates ${ }^{50,54}$. Such low-dose aerosol challenges have been developed for murine $M$. tuberculosis infection ${ }^{53}$ but are not widely used. Our observation that $\mathrm{CD} 4^{+}$ $\mathrm{T}$ cells are required for antibody efficacy may also mean that humanized mice ${ }^{55,56}$, or non-human primates whose immune systems would be better able to crosstalk with human antibodies, may be superior to inbred rodents for testing antibody efficacy. Coupling these approaches with culturing multiple clinical isolates of $M$. tuberculosis with intact capsule, which has been associated with increased protective responses ${ }^{3,34}$, may allow researchers to determine whether vaccine candidates can truly elicit sterilizing protective immunity.

\section{Can vaccination elicit protective antibody} responses? Generation of antibodies from natural infection and exposure and those elicited by immunization can be vastly different in terms of both function and ease of generation. Although the HIV experience may be useful in determining priorities for antibody research in TB, it also sounds a note of caution. Even with excellent in vitro correlates of protection and potent in vitro and in vivo efficacy of broadly neutralizing antibodies, eliciting such protective antibodies by immunization has been extremely problematic. The TB field has yet to pass the first two hurdles, but the urgency of the global TB problem has meant that more than a dozen clinical and hundreds of preclinical vaccine candidates have been proposed. Although the vast majority have focused on cell-mediated immunity, a number of candidates also elicit humoral responses $^{1,57}$. Other considerations are which models to test for protective antibody generation and efficacy? Even non-human primate models can be highly variable ${ }^{58}$. Increasingly, human challenge models are being used for testing vaccine efficacy ${ }^{59-62}$ and, although still some way off, are now starting to be developed for TB. Adjuvant choice also has a major impact on the quality and not just on the magnitude of the provoked immune response ${ }^{63-65}$, further complicating the number of parameters that need to be tested.

\section{Concluding remarks}

Re-examining humoral immunity to $\mathrm{TB}$ in the context of naturally arising human antibodies from individual donors has led to renewed interest in antibody-mediated immunity to $M$. tuberculosis infection, but this enthusiasm needs to be tempered with a sober realization of the many hurdles that need to be overcome before we can determine whether an antibody-based vaccine can be developed to prevent active TB. Development of vaccines that target both cell-mediated and humoral immunity ${ }^{57}$ may prove the most effective approach, with some already in late clinical trials ${ }^{66}$.

Are there interim milestones that can be tested more readily? Therapeutic antibodies are effective in HIV and other infections ${ }^{67}$, and killed whole-cell vaccines are being developed as adjuncts to antibiotic therapy in $\mathrm{TB}^{68}$. Such approaches are unlikely to be cost-effective for the treatment of the majority of TB cases - after all, TB is a disease of poverty and prevalent in resourcelimited settings. However, adjunctive therapy with therapeutic monoclonal antibodies may have a role in the treatment of extensively drug-resistant $\mathrm{TB}$, which is far more difficult and expensive to treat than standard TB cases. It would perhaps be fitting if the first clinical application of our recent advances in understanding M. tuberculosis antibody responses was the use of the 21 st century equivalent of serum therapy to fight the old enemy TB.
Hao Li and Babak Javid (iD *

Centre for Global Health and Infectious Diseases, Collaborative Innovation Centre for the Diagnosis and Treatment of Infectious Diseases, Tsinghua University School of Medicine, Beijing, China.

*e-mail: bjavid@gmail.com

https://doi.org/10.1038/s41577-018-0028-0

Published online 5 June 2018

1. Kaufmann, S. H. et al. Progress in tuberculosis vaccine development and host-directed therapies-a state of the art review. Lancet Respir. Med. 2, 301-320 (2014).

2. Chen, T. et al. Association of human antibodies to arabinomannan with enhanced mycobacterial opsonophagocytosis and intracellular growth reduction. J. Infecti. Diseases 214, 300-310 (2016).

3. $\mathrm{Li}, \mathrm{H}$. et al. Latently and uninfected healthcare workers exposed to TB make protective antibodies against Mycobacterium tuberculosis. Proc. Natl Acad. Sci. USA 114, 5023-5028 (2017).

4. Lu, L. L. et al. A functional role for antibodies in tuberculosis. Cell 167, 433-443.e414 (2016).

5. Zimmermann, N. et al. Human isotype-dependent inhibitory antibody responses against Mycobacterium tuberculosis. EMBO Mol. Med. 8, 1325-1339 (2016).

6. Mangtani, P. et al. Protection by BCG vaccine against tuberculosis: a systematic review of randomized controlled trials. Clin. Infect. Dis. 58, 470-480 (2014).

7. World Health Organization. Global Tuberculosis Report 2017. WHO http://www.who.int/tb/ publications/global_report/en/ (2017).

8. Casadevall, A. \& Scharff, M. D. Return to the past: the case for antibody-based therapies in infectious diseases. Clin. Infect. Dis. 21, 150-161 (1995).

9. Glatman-Freedman, A. \& Casadevall, A. Serum therapy for tuberculosis revisited: reappraisal of the role of antibody-mediated immunity against Mycobacterium tuberculosis. Clin. Microbiol. Rev. 11 514-532 (1998).

10. Cooper, A. M. Mouse model of tuberculosis. Cold Spring Harb. Perspect. Med. 5, a018556 (2015).

11. Havlir, D. V. \& Barnes, P. F. Tuberculosis in patients with human immunodeficiency virus infection N. Engl. J. Med. 340, 367-373 (1999).

12. Maglione, P. J., Xu, J. \& Chan, J. B cells moderate inflammatory progression and enhance bacterial containment upon pulmonary challenge with Mycobacterium tuberculosis. J. Immunol. 178, 7222-7234 (2007).

13. Vordermeier, H. M., Venkataprasad, N., Harris, D. P. \& Ivanyi, J. Increase of tuberculous infection in the organs of B cell-deficient mice. Clin. Exp. Immunol. 106, 312-316 (1996)

14. Phuah, J. Y., Mattila, J. T., Lin, P. L. \& Flynn, J. L. Activated $B$ cells in the granulomas of nonhuman primates infected with Mycobacterium tuberculosis. Am. J. Pathol. 181, 508-514 (2012)

15. Phuah, J. et al. Effects of B cell depletion on early Mycobacterium tuberculosis infection in cynomolgus macaques. Infect. Immun. 84, 1301-1311 (2016).

16. Achkar, J. M. \& Casadevall, A. Antibody-mediated immunity against tuberculosis: implications for vaccine development. Cell Host Microbe 13, 250-262 (2013).

17. Balu, S. et al. A novel human IgA monoclonal antibody protects against tuberculosis. J. Immunol. 186 3113-3119 (2011).

18. Buccheri, S. et al. Prevention of the postchemotherapy relapse of tuberculous infection by combined immunotherapy. Tuberculosis 89, 91-94 (2009).

19. Hamasur, B. et al. A mycobacterial lipoarabinomannan specific monoclonal antibody and its $F\left(a b^{\prime}\right)$ fragment prolong survival of mice infected with Mycobacterium tuberculosis. Clin. Exp. Immunol. 138, 30-38 (2004).

20. Pethe, K. et al. The heparin-binding haemagglutinin of M. tuberculosis is required for extrapulmonary dissemination. Nature 412, 190-194 (2001).

21. Teitelbaum, R. et al. A mAb recognizing a surface antigen of Mycobacterium tuberculosis enhances host survival. Proc. Natl Acad. Sci. USA 95, 15688-15693 (1998).

22. Guirado, E. et al. Passive serum therapy with polyclonal antibodies against Mycobacterium tuberculosis protects against post-chemotherapy relapse of tuberculosis infection in SCID mice. Microbes Infect. 8, 1252-1259 (2006). 
23. Roy, E. et al. Therapeutic efficacy of high-dose intravenous immunoglobulin in Mycobacterium tuberculosis infection in mice. Infect. Immun. 73, 6101-6109 (2005)

24. Forget, A., Benoit, J. C., Turcotte, R. \& Gusew-Chartrand, N. Enhancement activity of anti-mycobacterial sera in experimental Mycobacterium bovis (BCG) infection in mice. Infect. Immun. 13, 1301-1306 (1976).

25. Tameris, M. D. et al. Safety and efficacy of MVA85A, a new tuberculosis vaccine, in infants previously vaccinated with BCG: a randomised, placebo-controlled phase $2 \mathrm{~b}$ trial. Lancet 381 , 1021-1028 (2013)

26. Beveridge, N. E. et al. Immunisation with BCG and recombinant MVA85A induces long-lasting, polyfunctional Mycobacterium tuberculosis-specific $\mathrm{CD}^{+}$memory T lymphocyte populations. Eur. J. Immunol. 37, 3089-3100 (2007).

27. Beverley, P. Selective presentation of MVA85A tuberculosis booster vaccine preclinical animal data. Int J. Epidemiol. 45, 581-582 (2016).

28. Sakai, S. et al. CD4 T cell-derived IFN-gamma plays a minimal role in control of pulmonary Mycobacterium tuberculosis infection and must be actively repressed by PD-1 to prevent lethal disease. PLoS Pathog. 12 , e1005667 (2016).

29. Burton, D. R. \& Hangartner, L. Broadly neutralizing antibodies to HIV and Their role in vaccine design. Annu. Rev. Immunol. 34, 635-659 (2016).

30. Costello, A. M. et al. Does antibody to mycobacterial antigens, including lipoarabinomannan, limit dissemination in childhood tuberculosis? Trans. R. Soc Trop. Med. Hyg. 86, 686-692 (1992).

31. Chu, H. et al. Risk of tuberculosis among healthcare workers in an intermediate-burden country: a nationwide population study. J. Infect. 69, 525-532 (2014).

32. Zhou, F. et al. Latent tuberculosis infection and occupational protection among health care workers in two types of public hospitals in China. PLOS One 9 , e104673 (2014)

33. Pai, M. et al. Tuberculosis. Nat. Rev. Dis. Primers 2 , 16076 (2016).

34. Prados-Rosales, R. et al. The type of growth medium affects the presence of a mycobacterial capsule and is associated with differences in protective efficacy of BCG vaccination against Mycobacterium tuberculosis J. Infecti. Diseases 214, 426-437 (2016).

35. Cole, S. T. et al. Deciphering the biology of Mycobacterium tuberculosis from the complete genome sequence. Nature 393, 537-544 (1998).

36. Sani, M. et al. Direct visualization by cryo-EM of the mycobacterial capsular layer: a labile structure containing ESX-1-secreted proteins. PLoS Pathog. 6 e1000794 (2010).

37. Deng, J. et al. Mycobacterium tuberculosis proteome microarray for global studies of protein function and immunogenicity. Cell Rep. 9, 2317-2329 (2014).

38. Prados-Rosales, R. et al. Enhanced control of Mycobacterium tuberculosis extrapulmonary dissemination in mice by an arabinomannan-protein conjugate vaccine. PLoS Pathog. 13, e1006250 (2017).

39. Shin, H. J., Franco, L. H., Nair, V. R., Collins, A. C. \& Shiloh, M. U. A baculovirus-conjugated mimotope vaccine targeting Mycobacterium tuberculosis lipoarabinomannan. PLOS One 12, e0185945 (2017).

40. Bournazos, S. et al. Broadly neutralizing anti-HIV-1 antibodies require Fc effector functions for in vivo activity. Cell 158, 1243-1253 (2014).

41. Olivares, N. et al. The protective effect of immunoglobulin in murine tuberculosis is dependent on IgG glycosylation. Pathog. Dis. 69, 176-183 (2013).

42. Li, H., Wu, M., Shi, Y. \& Javid, B. Over-expression of the mycobacterial trehalose-phosphate phosphatase OtsB2 results in a defect in macrophage phagocytosis associated with increased mycobacterial-macrophage adhesion. Front. Microbiol. 7, 1754 (2016).

43. Bogaert, D., Sluijter, M., De Groot, R. \& Hermans, P. W. Multiplex opsonophagocytosis assay (MOPA): a useful tool for the monitoring of the 7-valent pneumococcal conjugate vaccine. Vaccine $\mathbf{2 2}$, 4014-4020 (2004).

44. Armstrong, J. A. \& Hart, P. D. Phagosome-lysosome interactions in cultured macrophages infected with virulent tubercle bacilli. Reversal of the usual nonfusion pattern and observations on bacterial survival. J. Exp. Med. 142, 1-16 (1975).

45. Kumar, S. K., Singh, P. \& Sinha, S. Naturally produced opsonizing antibodies restrict the survival of Mycobacterium tuberculosis in human macrophages by augmenting phagosome maturation. Open Biol. 5, 150171 (2015).

46. Wahid, R. et al. Live oral Salmonella enterica serovar Typhi vaccines Ty21a and CVD 909 induce opsonophagocytic functional antibodies in humans that cross-react with S. Paratyphi A and S. Paratyphi B. Clin. Vaccine Immunol. 21, 427-434 (2014).

47. McSorley, S. J. \& Jenkins, M. K. Antibody is required for protection against virulent but not attenuated Salmonella enterica serovar typhimurium. Infect. Immun. 68, 3344-3348 (2000).

48. van Els, C. et al. Fast vaccine design and development based on correlates of protection (COPs). Hum. Vaccin Immunother. 10, 1935-1948 (2014).

49. Corey, L et al. Immune correlates of vaccine protection against HIV-1 acquisition. Science Trans/ Med. 7, 310rv7 (2015)

50. Karp, C. L., Wilson, C. B. \& Stuart, L. M. Tuberculosis vaccines: barriers and prospects on the quest for a transformative tool. Immunol. Rev. 264, 363-381 (2015).

51. Su, H. W. et al. The essential mycobacterial amidotransferase GatCAB is a modulator of specific translational fidelity. Nat. Microbiol. 1, 16147 (2016)

52. Riley, R. L. et al. Aerial dissemination of pulmonary tuberculosis. A two-year study of contagion in a tuberculosis ward. 1959. Am. J. Epidemiol. 142, 3-14 (1995).

53. Saini, D. et al. Ultra-low dose of Mycobacterium tuberculosis aerosol creates partial infection in mice. Tuberculosis 92, 160-165 (2012).

54. Gautam, R. et al. A single injection of anti-HIV-1 antibodies protects against repeated SHIV challenges. Nature 533, 105-109 (2016).

55. Calderon, V. E. et al. A humanized mouse model of tuberculosis. PLOS One 8, e63331 (2013).

56. Grover, A. et al. Humanized NOG mice as a model for tuberculosis vaccine-induced immunity: a comparative analysis with the mouse and guinea pig models of tuberculosis. Immunology 152, 150-162 (2017).
57. Gengenbacher, M., Nieuwenhuizen, N. E. \& Kaufmann, S. BCG - old workhorse, new skills. Curr. Opin. Immunol. 47, 8-16 (2017).

58. Maiello, P. et al. Rhesus macaques are more susceptible to progressive tuberculosis than cynomolgus macaques: a quantitative comparison. Infect. Immun. 86, e00505-17 (2017).

59. Sauerwein, R. W., Roestenberg, M. \& Moorthy, V. S. Experimental human challenge infections can accelerate clinical malaria vaccine development. Nat. Rev. Immunol. 11, 57-64 (2011).

60. Collins, A. M. et al. First human challenge testing of a pneumococcal vaccine. Double-blind randomized controlled trial. Am. J. Respiratory Crit. Care Med. 192, 853-858 (2015)

61. Dobinson, H. C. et al. Evaluation of the clinical and microbiological response to Salmonella Paratyphi A infection in the first paratyphoid human challenge model. Clin. Infect. Dis. 64, 1066-1073 (2017).

62. Kirkpatrick, B. D. et al. The live attenuated dengue vaccine TV003 elicits complete protection against dengue in a human challenge model. Sci. Transl Med. 8, 330ra336 (2016).

63. Garcon, N., Heppner, D. G. \& Cohen, J. Development of RTS,S/AS02: a purified subunit-based malaria vaccine candidate formulated with a novel adjuvant. Expert Rev. Vaccines 2, 231-238 (2003).

64. Ackerman, M. E., Barouch, D. H. \& Alter, G. Systems serology for evaluation of HIV vaccine trials. Immunol. Rev. 275, 262-270 (2017).

65. Vaccari, M. et al. Adjuvant-dependent innate and adaptive immune signatures of risk of SIVmac251 acquisition. Nature Med. 22, 762-770 (2016).

66. Nieuwenhuizen, N. E. et al. The recombinant Bacille Calmette-Guerin vaccine VPM1002: ready for clinical efficacy testing. Front. Immunol. 8, 1147 (2017).

67. Salazar, G., Zhang, N., Fu, T. M. \& An, Z. Antibody therapies for the prevention and treatment of viral infections. NPJ Vaccines 2, 19 (2017).

68. Cardona, P. J. The progress of therapeutic vaccination with regard to tuberculosis. Front. Microbiol. 7, 1536 (2016).

\section{Acknowledgements}

This work was in part funded by a grant from the National Science Foundation of China (81661128044) to B.J. B.J. is a Wellcome Trust Investigator.

\section{Author contributions}

Both authors contributed to researching data, discussion of content and the writing of the article. B.J. reviewed and edited the manuscript before submission.

\section{Competing interests}

The authors declare no competing interests.

\section{Publisher's note}

Springer Nature remains neutral with regard to jurisdictional claims in published maps and institutional affiliations.

\section{Reviewer information}

Nature Reviews Immunology thanks S. Kaufmann and the other, anonymous reviewer(s) for their contribution to the peer review of this work. 